\title{
The interaction of HIV-1 and HIV-2 with cellular protein trafficking pathways
}

\author{
Justine Alford, Robert Spooner, Michela Marongiu, Emma Anderson* \\ From Frontiers of Retrovirology: Complex retroviruses, retroelements and their hosts \\ Cambridge, UK. 16-18 September 2013
}

\section{Background}

Assembly and release of HIV-1 particles occurs at the plasma membrane and is dependent on trafficking of Gag from sites of protein synthesis to sites of particle assembly. For example, the interaction of HIV-1 Gag with components of cellular trafficking pathways such as clathrin adaptor proteins (AP) 1 and 3 has been shown to be required for HIV-1 virion production $[1,2]$. This is likely to place a large burden on cellular trafficking pathways, but this has not previously been investigated. We addressed the questions of whether HIV-1 disrupts cellular protein trafficking and whether HIV-2 has the same effect.

\section{Materials and methods}

We used the well characterised pathways of diphtheria toxin (DTx) and ricin toxin (RTx) trafficking to measure the effect of HIV-1 and HIV-2 on toxin-induced inhibition of protein synthesis. HIV-1 and -2 Gag localisation, and the role of AP-3 in Gag trafficking, was studied using confocal immunofluorescence microscopy, siRNAmediated knockdown of AP-3, and virion release assays.

\section{Results}

HIV-1 had a protective effect against DTx-, but not RTxinduced cytotoxicity, demonstrating that endosomal trafficking is specifically disrupted in the presence of HIV-1. However, HIV-2 had a significantly higher protective effect against DTx, suggesting greater disruption of endosomal trafficking by HIV-2. In agreement with these results, we observed a much higher proportion of HIV-2 Gag localised to endosomal compartments than HIV-1 Gag. There was strong co-localisation between HIV-2 Gag, AP-3 and clathrin, but less so with HIV-1 Gag. siRNA-mediated knockdown of AP-3 resulted in an increase in the proportion of both HIV-1 and HIV-2 Gag localised to endosomal compartments, a decrease in the proportion at the plasma membrane, and a reduction in virion release.

\section{Conclusions}

HIV-1 and HIV-2 both utilise cellular protein trafficking pathways, which disrupts normal cell functioning. HIV-2 has a particularly disruptive effect on the endosomal pathway, and we observed that HIV-2 Gag accumulates in endosomal compartments. AP-3 and clathrin are recruited to these compartments in the presence of HIV-2, and we hypothesise that this is a requirement for transport of Gag and/or virions from endosomes to the plasma membrane.

\section{Acknowledgements}

This work was funded by the MRC

Published: 19 September 2013

\section{References}

1. Camus G, Segura-Morales C, Molle D, Lopez-Verges S, Beon-Psecia C, Cazevieille C, Schu P, Bertrand E, Berlioz-Torrent C, Basyuk E: The clathrin adaptor complex AP-1 binds HIV-1 and MLV Gag and facilitates their budding. Mol Biol Cell 2007, 18:3193-3203.

2. Dong X, Li H, Derdowski A, Ding L, Burnett A, Chen X, Peters TR, Dermody TS, Woodruff E, Wang JJ, Spearman P: AP-3 directs the intracellular trafficking of HIV-1 Gag and plays a key role in particle assembly. Cell 2005, 120:663-674.

doi:10.1186/1742-4690-10-S1-P2

Cite this article as: Alford et al:: The interaction of HIV-1 and HIV-2 with cellular protein trafficking pathways. Retrovirology 2013 10(Suppl 1):P2. 